\title{
Volatile Profiling Aided in the Isolation of Anti-Proliferative Lupeol from the Roots of Clinacanthus nutans (Burm. f.) Lindau
}

\author{
Angelina Ying Fang Cheng ${ }^{1}$, Peik Lin Teoh ${ }^{1} \mathbb{D}$, Lalith Jayasinghe ${ }^{2}$ and Bo Eng Cheong ${ }^{1, * \mathbb{D}}$ \\ 1 Biotechnology Research Institute, Universiti Malaysia Sabah, Jalan UMS, Kota Kinabalu 88400, Malaysia; \\ angelinacheng16@gmail.com (A.Y.F.C.); peiklin@ums.edu.my (P.L.T.) \\ 2 National Institute of Fundamental Studies, Hantana Road, Kandy 20000, Sri Lanka; lalith.ja@nifs.ac.lk \\ * Correspondence: becheong@ums.edu.my; Tel.: +60-88-320000 (ext. 8530)
}

check for updates

Citation: Cheng, A.Y.F.; Teoh, P.L.; Jayasinghe, L.; Cheong, B.E. Volatile Profiling Aided in the Isolation of Anti-Proliferative Lupeol from the Roots of Clinacanthus nutans (Burm. f.) Lindau. Processes 2021, 9, 1383. https://doi.org/10.3390/pr9081383

Academic Editor: Zongbi Bao

Received: 29 June 2021

Accepted: 30 July 2021

Published: 9 August 2021

Publisher's Note: MDPI stays neutral with regard to jurisdictional claims in published maps and institutional affiliations.

Copyright: (c) 2021 by the authors. Licensee MDPI, Basel, Switzerland. This article is an open access article distributed under the terms and conditions of the Creative Commons Attribution (CC BY) license (https:// creativecommons.org/licenses/by/ $4.0 /)$.

\begin{abstract}
Isolation of anti-proliferative compounds from plants is always hindered by the complexities of the plant's nature and tedious processes. Clinacanthus nutans (Burm. f.) Lindau is a medicinal plant with reported anti-proliferative activities. Our study aimed to isolate potential anti-proliferative compounds present in C. nutans plant. To start with, for our study, we came up with a strategy by first profiling the volatile compounds present in the leaf, stem and root of C. nutans using GC-MS. Comparing the plant's volatile profiles greatly narrowed down our target of study. We decided to start with the isolation and characterization of a pentacyclic terpenoid, i.e., lupeol from the roots of C. nutans, as this compound was found to present abundantly in the roots compared to the leaf or stem. We developed a simple maceration and re-crystallization method, without the necessity to go through the fractionation or column chromatography for the isolation of lupeol. Characterizations of the isolated compound identified the compound as lupeol. The anti-proliferative activity of the isolated lupeol was further investigated against the MCF-7 cell line, which showed comparable anti-proliferative activity with the authentic lupeol and camptothecin. Our strategy to profile every part of the plant first, followed by selection of the most suitable plant part and targeted compound proved useful for further isolation and characterization bioactive compound from C. nutans.
\end{abstract}

Keywords: Clinacanthus nutans; profiling; root; GC-MS; lupeol; anti-proliferative; MCF-7 cells

\section{Introduction}

Clinacanthus nutans (Burm. f.) Lindau from the Acanthaceae family is a small shrub which is naturally found in tropical and subtropical Asian countries, including Malaysia, Thailand, Indonesia, Vietnam, and China [1]. In Malaysia, the plant is commonly known as Belalai Gajah and Sabah snake grass [2]. The aerial part of this plant, i.e., the leaf (Figure 1) has been shown to possess a vast spectrum of pharmacological activities, in which the antiproliferative activities on various human cancer cell lines have been extensively studied. For instance, C. nutans leaf extracted with chloroform, methanol, and water has been reported to demonstrate anti-proliferative effects against various human cancer cell lines, such as the liver hepatocellular carcinoma cell line (HepG2), neuroblastoma cell line (IMR32), lung cancer cell line (NCI-H23), gastric cancer cell line (SNU-1), colon adenocarcinoma cell line (LS-174T), erythroleukemia cell line (K-562), human cervical cancer cell line (HeLa), and Burkitt's lymphoma cell line (Raji) [3]. On the other hand, the leaf extracted with ethanol and ethyl acetate has also been reported to exhibit strong cytotoxic effects against the estrogen-dependent human breast cancer cell line, MCF-7 [4]. Meanwhile, Fong and colleagues have collected C. nutans leaves from different countries, such as Malaysia, Thailand and Vietnam. They extracted the collected leaves with methanol and found that the leaf extracts possess cytotoxicity and apoptotic activities against the human skin cancer cell line, D24 melanoma cells [5]. In addition to the anti-proliferative effects on various cancer cell lines, the aerial parts of the plant have also been reported to exhibit significant antitumor and apoptotic activities on the in vivo mice cancer model [6]. While 
most of the anticancer studies on this plant are focused on the aerial part, our group have conducted an anti-proliferative study on the underground part, i.e., the root (Figure 1) [7]. The crude methanolic and ethyl acetate extracts of $C$. nutans roots were found to exhibit anti-proliferative and apoptotic effects on breast cancer (MCF-7) and cervical cancer (HeLa) cell lines [Teoh].

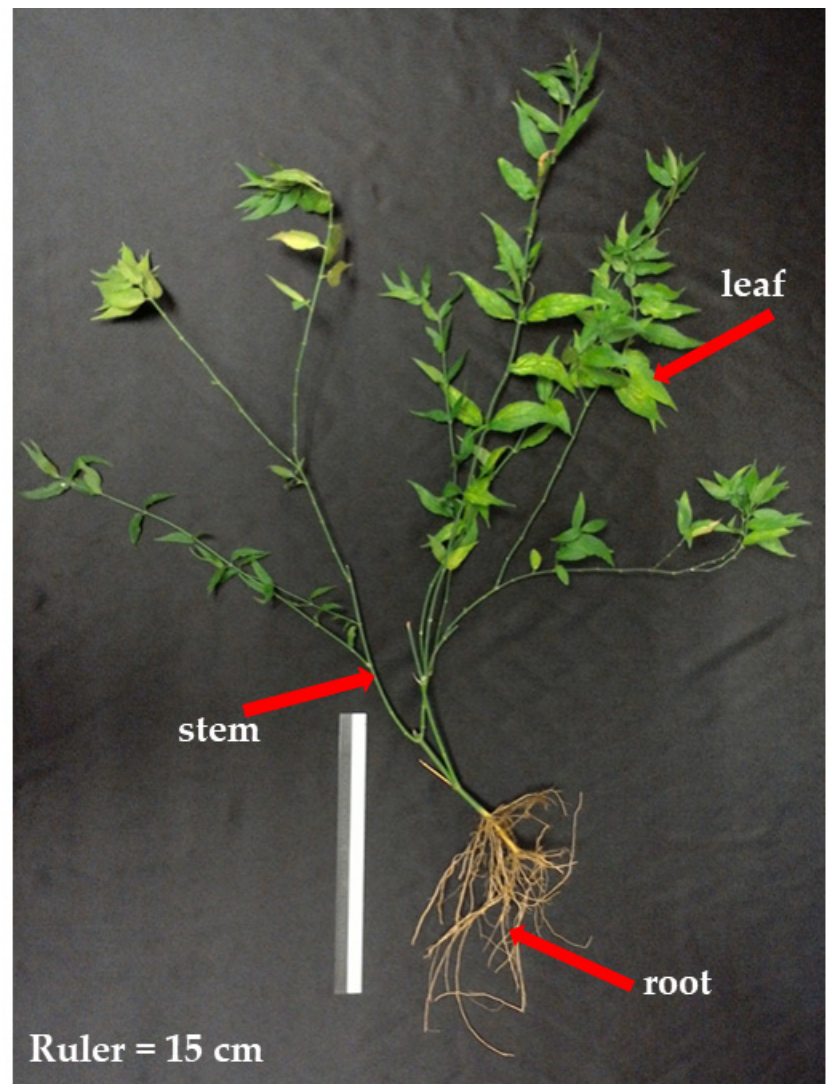

Figure 1. A Clinacanthus nutans plant. The arrows show the leaf, stem and root parts of the plant used for extraction and volatile profiling in this study. The roots were further used for the isolation and characterization of lupeol.

Owing to its promising anti-proliferative activities, several attempts have been conducted to isolate the anti-proliferative compounds and/or active fractions from the C. $n u$ tans leaf. Huang and colleagues [6] have extracted the aerial parts of $C$. nutans with ethanol, followed by further fractionation on a Diaion HP-20 macroporous adsorption resin to produce a 30\% ethanol fraction (CN30) and other fractions. The CN30 fraction was found to be able to induce inhibition in tumor size and weight of the HepA xenograft model mouse (hepatoma cell-injected mice). The active components in $\mathrm{CN} 30$ were therefore further fractionated and purified. High-performance liquid chromatography (HPLC) and mass spectrometry (LC/MS/MS) analyses revealed that there were seven active compounds present in $\mathrm{CN} 30$. The seven identified active components were shaftoside, apigenin $6,8-C-\alpha$-L-pyranarabinoside, orientin, isoorientin, vitexin, isovitexin, and gallic acid. After that, the same research group conducted another study to isolate a novel polysaccharidepeptide complex, which they named CNP-1-2, by a series of complex purification methods from C. nutans leaves [8]. This CNP-1-2 complex was found to show the strongest growth inhibition effect on the human gastric cancer cell line (SGC-7901) and was able to stimulate the activation of macrophages. On the other hand, another research group led by Mutazah and colleagues [9] has managed to fractionate two active fractions from the bark powder of C. nutans. These two fractions, which they named A12 and A17, were found to contain 
sulfur-containing compounds, including entadamide $C$ and clinamide $D$, that possessed strong cytotoxic activities against MDA-MB-231 and MCF-7 breast cancer cell lines.

From the studies above, it can be postulated that the isolation of anti-proliferative compounds from C. nutans is not easy. This may be due to the complexities of the plant's nature, and also various fractionation/chromatographic steps which need to be optimized. Our study therefore aimed to pre-screen (profile) the volatile compounds present in every part of the plants first, followed by the selection and isolation of target compound. The details of the study are described in the subsequent sections.

\section{Materials and Methods}

\subsection{Solvents, Chemicals and Cell Lines}

All the solvents used in this study were of analytical and/or high performance liquid chromatography (HPLC) grade, and purchased from Fisher Scientific (Pittsburgh, PA, USA). The solvents used were methanol, ethyl acetate and dimethylsulfoxide (DMSO). The authentic reference standard, lupeol (L5632-25 mg, $\geq 94 \%$ purity) for characterization purposes was obtained from Sigma-Aldrich (St. Louis, MO, USA). Roswell Park Memorial Institute (RPMI) 1640, Phosphate-Buffered Saline (PBS), $2.5 \mathrm{~g} / \mathrm{L}$ trypsin-1mmol/L EDTA, and $0.5 \%$ trypan blue stain solution were purchased from Nacalai Tesque (Kyoto, Japan), whereas the Fetal Bovine Serum (FBS) was purchased from JR Scientific (Woodland, CA, USA). The cell proliferation kit was purchased from Roche Diagnostics (Mannheim, Germany).

\subsection{Plant Materials}

Clinacanthus nutans (Burm. f.) Lindau plants were purchased from herbal suppliers at a local market in Kota Kinabalu, Sabah, Malaysia. The plants were verified by a botanist from the Faculty of Science and Natural Resources, Assoc. Prof. Dr. Berhaman Ahmad, and a voucher specimen (ACCN 001/2013) was deposited in the herbarium of Universiti Malaysia Sabah. The plants were first cleaned thoroughly with tap water to remove the dirt, followed by rinsing several times with distilled water and dried with a paper towel. The leaves, stems and roots were excised from the plants, freeze-dried for five days and ground into powder using a heavy-duty blender. The plant samples in powder form were stored at $-80^{\circ} \mathrm{C}$ until further analysis. Some of the stems of the purchased plants were re-grown in soil for a duration of 6 months for photo shooting purposes.

\subsection{Extraction of C. nutans Leaf, Stem, and Root for Volatile Profiling}

Approximately $0.5 \mathrm{~g}$ of each freeze-dried C. nutans leaf, stem, and root sample was weighed and added with $10 \mathrm{~mL}$ of absolute ethyl acetate, followed by maceration at $37^{\circ} \mathrm{C}$ with $180 \mathrm{rpm}$ agitation for three days in dark conditions. After three days of extraction, the plant extracts were filtered with Whatman filter paper, followed by drying under vacuum pressure by using a rotary evaporator at $40{ }^{\circ} \mathrm{C}$, and then stored at $-80{ }^{\circ} \mathrm{C}$ until further analysis. For each plant's part, the whole extraction process was repeated another two times to produce three replicates $(n=3)$ per leaf, stem and root.

\subsection{Volatile Profiling of the Crude Leaf, Stem, and Root Extracts Using GC-MS}

The crude C. nutans leaf, stem, and root ethyl acetate extracts were re-dissolved in HPLC-grade ethyl acetate to appropriate concentrations and filtered using $0.22 \mu \mathrm{m}$ syringe filters prior to GC-MS analyses. The GC-MS analysis was carried out according to [9], with some modifications. Briefly, $1 \mu \mathrm{L}$ aliquot of each sample was injected into the GC-MS equipment (GC model 7890 and MS model 5975C; from Agilent Technologies, Santa Clara, CA, USA). An HP- $5 \mathrm{~ms}$ capillary column $(30 \mathrm{~m} \times 0.250 \mathrm{~mm}$ inner diameter $\times 0.25 \mu \mathrm{m}$ of film thickness) from Agilent Technologies was used for separation. GC-MS was performed in a splitless mode and pure helium was used as the carrier gas with a flow rate of $1 \mathrm{~mL} / \mathrm{min}$. The MS source was pre-set to $230^{\circ} \mathrm{C}$ and MS quad temperature was recorded at $150{ }^{\circ} \mathrm{C}$. The injector temperature was set to $250^{\circ} \mathrm{C}$ with an injection volume of $1 \mu \mathrm{L}$ per sample. The 
oven temperature was programmed from $220^{\circ} \mathrm{C}$ for $10 \mathrm{~min}$ and continuously increased at a rate of $5{ }^{\circ} \mathrm{C} / \mathrm{min}$ until a temperature of $300{ }^{\circ} \mathrm{C}$ was reached and then retained for $10 \mathrm{~min}$. The total run time was $36 \mathrm{~min}$. The post-run was fixed at $200{ }^{\circ} \mathrm{C}$ for $5 \mathrm{~min}$, and the ion source temperature was constant at $200{ }^{\circ} \mathrm{C}$. The mass analyzer was performed in a full scan mode from $\mathrm{m} / \mathrm{z} 40$ to 550 , and mass spectra were obtained at EI $=70 \mathrm{eV}$. The identification of the compounds was performed by spectra matching using the National Institute Standard and Technology (NIST) library version 11 (NIST11). The leaf, stem, and root profiles obtained were compared to select for the most suitable plant's part to be used for the subsequent isolation of target compound.

\subsection{Extraction of C. nutans Root for the Isolation of Lupeol}

The selected plant part in this study was the roots, and the target compound was lupeol. First of all, $80 \mathrm{~g}$ of freeze-dried C. nutans roots were weighed and added into a conical flask containing $200 \mathrm{~mL}$ of absolute ethyl acetate. The extract was put inside a rotary shaker and macerated at $37^{\circ} \mathrm{C}$ with $180 \mathrm{rpm}$ agitation for a consecutive five days in dark conditions. After five days of extraction, the crude root extract was filtered with Whatman filter paper, transferred to a new flask, and stored at $4{ }^{\circ} \mathrm{C}$. A repeated extraction was performed on the remaining root residue in the extraction flask with the addition of another $200 \mathrm{~mL}$ of ethyl acetate but only for three days. The second crude root extract was filtered and combined with the first extract, followed by drying under vacuum pressure using a rotary evaporator at $40^{\circ} \mathrm{C}$ and stored at $-80^{\circ} \mathrm{C}$ prior to the recrystallization step.

\subsection{Recrystallization of C. nutans Root Extract for the Isolation of Lupeol}

The dried C. nutans crude root extract in Part 2.5 above was re-dissolved in $100 \mathrm{~mL}$ of absolute methanol and added into an Erlenmeyer flask, followed by heat-stirring on a hot plate until all the extract was fully dissolved and the methanol solvent boiled. The Erlenmeyer flask was then left to cool down inside a huge glass beaker filled with ice. While cooling down, the inner side of the Erlenmeyer flask was gently stirred by using a glass rod to induce crystal formation (nucleation). After ten minutes, the formed crystals were filtered out from the methanol solvent using a Buchner funnel under vacuum pressure. The filtered crystals were collected and left to dry inside a laminar flow for several minutes until a white amorphous powder was obtained. The collected white amorphous powder was weighed, followed by identification and characterization using various analytical instruments.

\subsection{GC-MS Analyses and Comparisons of the Isolated and Authentic Reference Standard Lupeols}

The isolated white amorphous compound from $C$. nutans roots and the purchased authentic standard lupeol were dissolved in HPLC-grade ethyl acetate to appropriate concentrations and were filtered using $0.22 \mu \mathrm{m}$ syringe filters prior to GC-MS analyses. The GC-MS analysis was carried out according to Part 2.4, with some modifications on the ramping program. Briefly, the oven temperature was programmed from $220^{\circ} \mathrm{C}$ for $10 \mathrm{~min}$ and continuously increased at a rate of $5{ }^{\circ} \mathrm{C} / \mathrm{min}$ until a temperature of $300{ }^{\circ} \mathrm{C}$ was reached and then retained for $10 \mathrm{~min}$. The total run time was $41 \mathrm{~min}$. The identification of the compound was determined using the NIST11 library (Cas number for lupeol is 000545-47-1, entry number in library is \#221171) and also compared with the MS spectra of the reference standard lupeol. In addition, further confirmation of the identity and purity of lupeol isolated from C. nutans roots was carried out by spiking the standard lupeol into the isolated lupeol sample, followed by a re-analysis with GC-MS to observe if only one overlapped peak (lupeol) was present in the chromatogram or not.

\subsection{Characterization of the Isolated Lupeol from C. nutans Roots}

Firstly, the melting point of the isolated lupeol was determined with SMP40 Automatic Melting Point Apparatus (Stuart, UK). Next, the infrared (IR) spectra of the isolated and standard lupeol were determined using a Cary 630 FTIR Spectrometer (Agilent Technolo- 
gies, CA, USA). Finally, structural elucidation of the isolated compound was determined using 1D-FT-NMR in $600 \mathrm{MHz}$ (Bruker, Germany) for both ${ }^{1} \mathrm{H}-\mathrm{NMR}$ and ${ }^{13} \mathrm{C}-\mathrm{NMR}$.

\subsection{Anti-Proliferative Activities of the Isolated Lupeol Compared to the Authentic Standard Lupeol}

The anti-proliferative activities of isolated and standard lupeol were determined using MTT assay, which was performed using the Cell Proliferation Kit I (Roche Diagnostics, Mannheim, Germany) according to the manufacturer's protocol. Human breast cancer (MCF-7) cell line was seeded in a 96-well plate $\left(3 \times 10^{4}\right.$ cells/well) with $100 \mu \mathrm{L}$ of RPMI 1640 medium and incubated at $37^{\circ} \mathrm{C}$ with $5 \% \mathrm{CO}_{2}$ of humidified atmosphere overnight. After $24 \mathrm{~h}$, cells were treated with different concentrations of the isolated compound and standard lupeol ranging from 10 to $50 \mu \mathrm{g} / \mathrm{mL}$ for $72 \mathrm{~h}$. For positive control, cells were treated with camptothecin at the concentrations of $0.5-2.0 \mu \mathrm{g} / \mathrm{mL}$ for $72 \mathrm{~h}$. Cells treated with DMSO were used as a control. The cell proliferation was measured at $570 \mathrm{~nm}$ using a microplate reader (Molecular Devices, Sunnyvale, CA, USA). The $\mathrm{IC}_{50}$ values ( $50 \%$ growth inhibitory concentration) were calculated from three independent experiments, in which there were three technical replicates $(n=3)$ per concentration per experiment.

\subsection{Statistical Analysis}

The $\mathrm{IC}_{50}$ values of the isolated and standard lupeol were expressed as mean $\pm \mathrm{SD}$ of the three independent experiments $(n=3)$, which were calculated using a non-linear dose-response curve fitting analysis with GraphPad Prism 7 (GraphPad Software, Inc., San Diego, CA, USA). The level of significance $\left({ }^{*} p<0.05,{ }^{* *} p<0.01\right.$ and $\left.{ }^{* * *} p<0.001\right)$ was determined using one-way ANOVA followed by a post hoc Dunnett test by comparison to the untreated controls.

\section{Results}

3.1. Volatile Profiling of C. nutans Reveals Different Accumulations of Bioactive Metabolites in Different Parts of the Plant

In our volatile profiling of different parts of the $C$. nutans plant, ethyl acetate was used as the extraction solvent to extract for volatile or nonpolar compounds, and a GC-MS method, which we have optimized for the detection of volatiles or nonpolar compounds [7], was employed in this study. From the results, it was interesting to observe that the leaves, stems, and roots have different volatile profiles among each other (Figure 2). The leaves were found to contain vitamin $\mathrm{E}$ ( $\alpha$-tocopherol) as the most abundant compound, followed by squalene (the precursor of pentacyclic triterpenoids and phystosterols), and phytosterols, such as stigmasterol, $\beta / \gamma$-sitosterol, and campesterol. Meanwhile, the stems were shown to contain lupeol, phytosterols, squalene, and vitamin E ( $\alpha$-tocopherol). The most interesting part was found in the roots, where there was only one main peak present in the profile, which was designated as lupeol by the NIST11 library (with matched quality of 76). The other compounds which were observed in the leaves or stems were found to be very scarce in the root part. As lupeol has been reported to possess anti-proliferative activities (which will be discussed in the Discussion Section 4) and also the volatile profile of the root was simple, we then decided to make this compound our target in this study. 


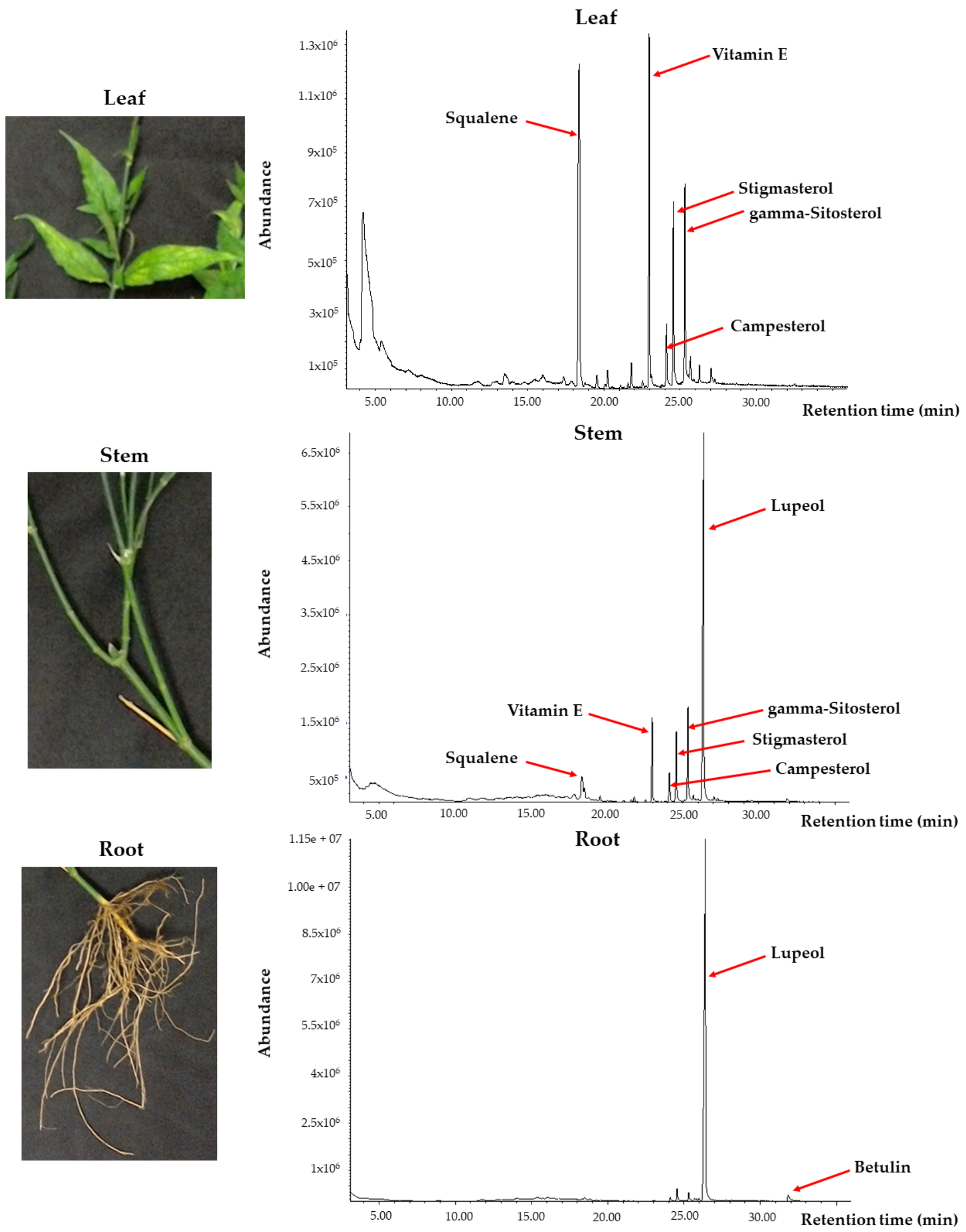

Figure 2. Volatile profiles of the leaf, stem, and root of C. nutans obtained using GC-MS analysis. 


\subsection{GC-MS Analysis Reveals the Isolation of Lupeol from C. nutans Roots}

Through maceration and recrystallization methods, $1.138 \mathrm{~g}$ of the white amorphous compound was successfully isolated from an initial $80 \mathrm{~g}$ of freeze-dried C. nutans roots. The extraction yield was therefore $1.42 \%(1.138 \mathrm{~g} / 80 \mathrm{~g} \times 100 \%)$, or $14.2 \mathrm{mg} / \mathrm{g}$ freeze-dried root. The white amorphous compound was then re-dissolved with HPLC-grade ethyl acetate and analyzed with GC-MS. The profile was compared with standard lupeol and isolated compound spiked with standard lupeol (Figure 3). From Figure 3A, we observed that only one single main peak was shown in the profile at a retention time of $28.769 \mathrm{~min}$ for the isolated white amorphous compound. This isolated compound also showed almost the same retention time with the purchased authentic reference standard, lupeol, which has an Rt of 28.789 min (Figure 3B). When the electron ionization-mass spectrum (EIMS) of the single peak from Figure 3A was compared to the EI-MS of standard lupeol, they were identical (Supplemental Figure S1A). When this isolated white amorphous compound was spiked with an equal amount of standard lupeol and loaded into GCMS, only one single peak was detected in the profile with a retention time of $28.799 \mathrm{~min}$ (Figure 3C). The EI-MS also overlapped well (Supplementary Figure S1A). Lupeol has a molecular weight of $426.729 \mathrm{~g} / \mathrm{mol}$ and a molecular formula of $\mathrm{C}_{30} \mathrm{H}_{50} \mathrm{O}$. The EI-MS of this single peak showed the presence of the parent ion (M+) at $m / z 426$, which corresponds to the molecular formula of $\mathrm{C}_{30} \mathrm{H}_{50} \mathrm{O}$ and most probably represented lupeol. The EIMS also revealed the presence of fragment ions at $m / z 189$ and 218, which were the main characteristic of a pentacyclic triterpenoid [10]. Another key feature of pentacyclic triterpenoid is the isopropenyl fragment at $m / z 411\left[\mathrm{M}-\mathrm{CH}_{3}\right]^{+}[11]$, which was also detected (Supplemental Figure S1A). Overall, the identical fragmentation patterns of EI-MS of the isolated compound with the fragmentation patterns of EI-MS of standard lupeol suggested that the isolated compound from C. nutans roots was lupeol.

\subsection{Characterization and Structural Elucidation of the Isolated Lupeol}

Characterizarion of the isolated white amorphous showed that it has a melting point of $216^{\circ} \mathrm{C}$. Meanwhile, the IR spectrum of the isolated compound was also studied (Supplemental Figure S2). The hydroxyl group $(-\mathrm{O}-\mathrm{H})$ vibration was shown as a broad intense band at $3303 \mathrm{~cm}^{-1}$, and a moderately intense band was observed at $1187 \mathrm{~cm}^{-1}$. The stretching and bending of the methyl group $\left(-\mathrm{C}-\mathrm{H}_{3}\right)$ were detected at $2930 \mathrm{~cm}^{-1}$ with an intense band and a moderately intense band at $1450 \mathrm{~cm}^{-1}$. In addition, the vibration of the $\left(-\mathrm{C}-\mathrm{H}_{2}\right)$ methylenic part was observed at $2861 \mathrm{~cm}^{-1}$, which displayed a weaker intense band. The vibration of the $(-\mathrm{C}=\mathrm{O})$ of the carboxylic acid group was also observed as an intense band at $1737 \mathrm{~cm}^{-1}$. At the wavelength of $1379 \mathrm{~cm}^{-1}$, there was the presence of $(-C-C)$ vibration of the methyl group as a moderate intense band. The out of the plane $(-\mathrm{C}-\mathrm{H})$ vibration was observed at $886 \mathrm{~cm}^{-1}$ as a strong intense band. Those obtained IR spectra data were in good agreement with the IR spectra of lupeol, as studied and reported by $[12,13]$.

Elucidation of the structure of the isolated compound was performed using ${ }^{1} \mathrm{H}-\mathrm{NMR}$ (600 MHz) and ${ }^{13} \mathrm{C}-\mathrm{NMR}(150 \mathrm{MHz})$. The ${ }^{1} \mathrm{H}-\mathrm{NMR}$ spectrum of the compound showed the presence of seven methyl groups at $\delta 0.98\left(\mathrm{~s}, \mathrm{H}_{3}-23\right), 0.79\left(\mathrm{~s}, \mathrm{H}_{3}-24\right), 0.83\left(\mathrm{~s}, \mathrm{H}_{3}-25\right), 1.03$ (s, $\left.\mathrm{H}_{3}-26\right), 1.03\left(\mathrm{~s}, \mathrm{H}_{3}-27\right), 0.81\left(\mathrm{~s}, \mathrm{H}_{3}-28\right)$, and $1.66\left(\mathrm{~s}, \mathrm{H}_{3}-30\right)$. The predominant characteristic of the lupane class of triterpenoid was found as a pair of singlets at $\delta 4.57$ and $\delta 4.69$ with ${ }^{1} \mathrm{H}$ for each singlet due to the terminal isopropenyl moiety or the vinylic proton located at carbon 29. Meanwhile, the presence of a doublet with one proton intensity found at $\delta 3.18$ $(\mathrm{dd}, \mathrm{J}=4.8 \mathrm{~Hz}, \mathrm{H}-3)$ was caused by the proton attached to the carbon bearing the hydroxyl group or an oxymethine proton located at C-3, which is identical as reported [10]. There was also one proton with a doublet of the triplet located at $\delta 2.37$, which was assigned to $19 \beta-H$ orientation, similar to the reported literature data [14]. The ${ }^{1} \mathrm{H}-\mathrm{NMR}$ spectrum for the isolated compound can be found in Supplemental Data S1A. 


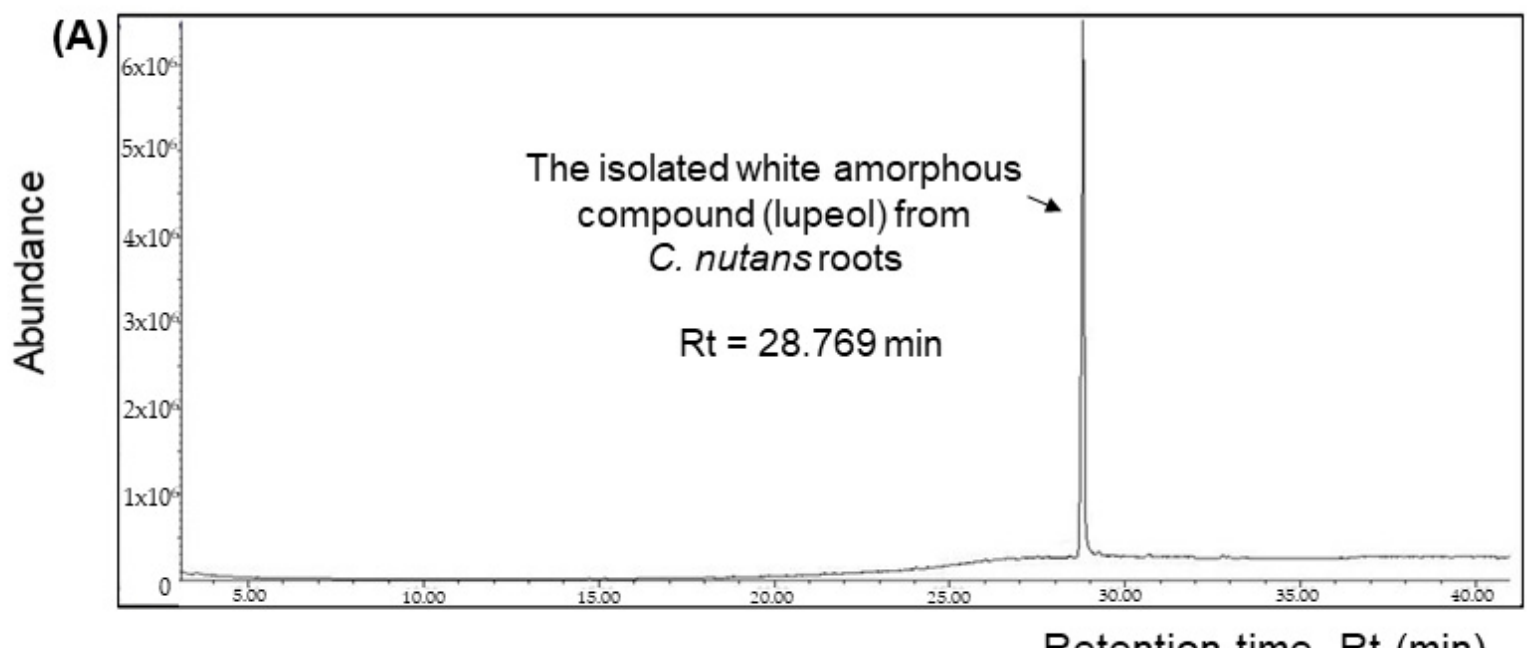

Retention time, Rt (min)

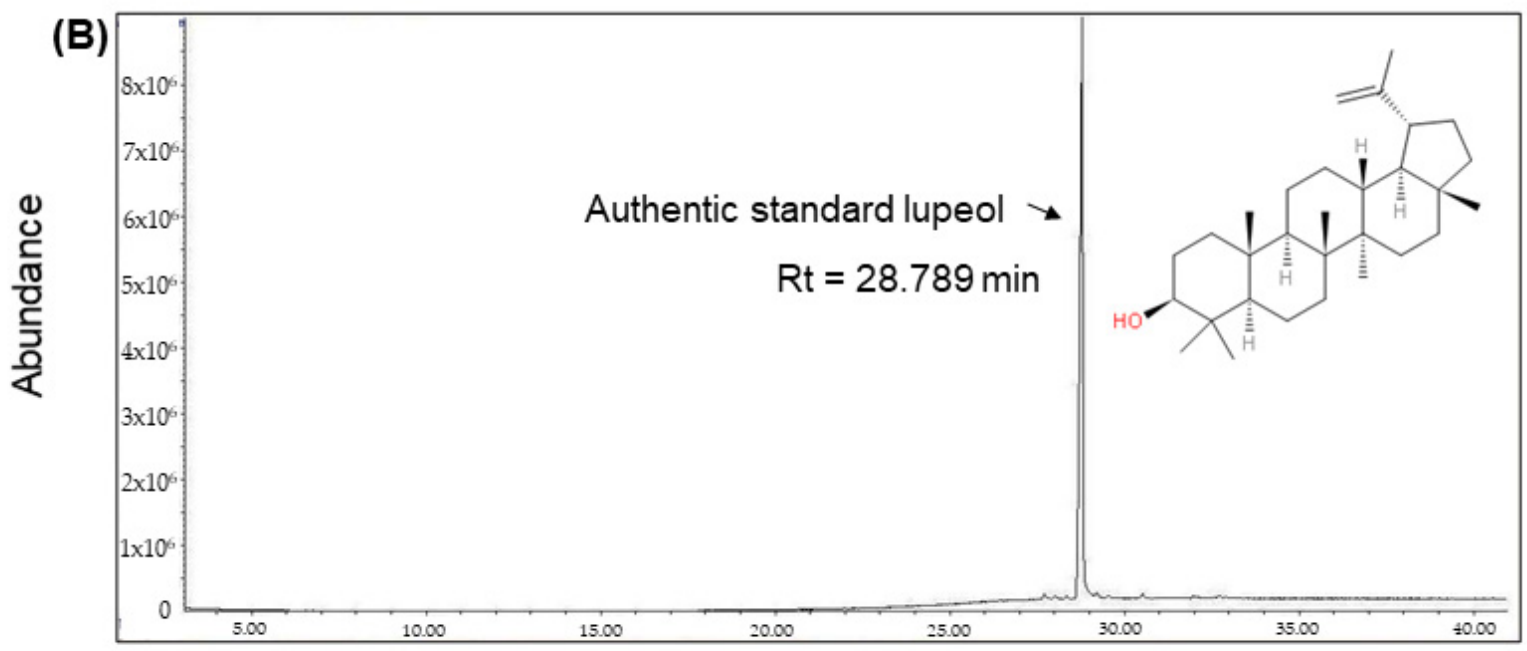

Retention time, Rt (min)

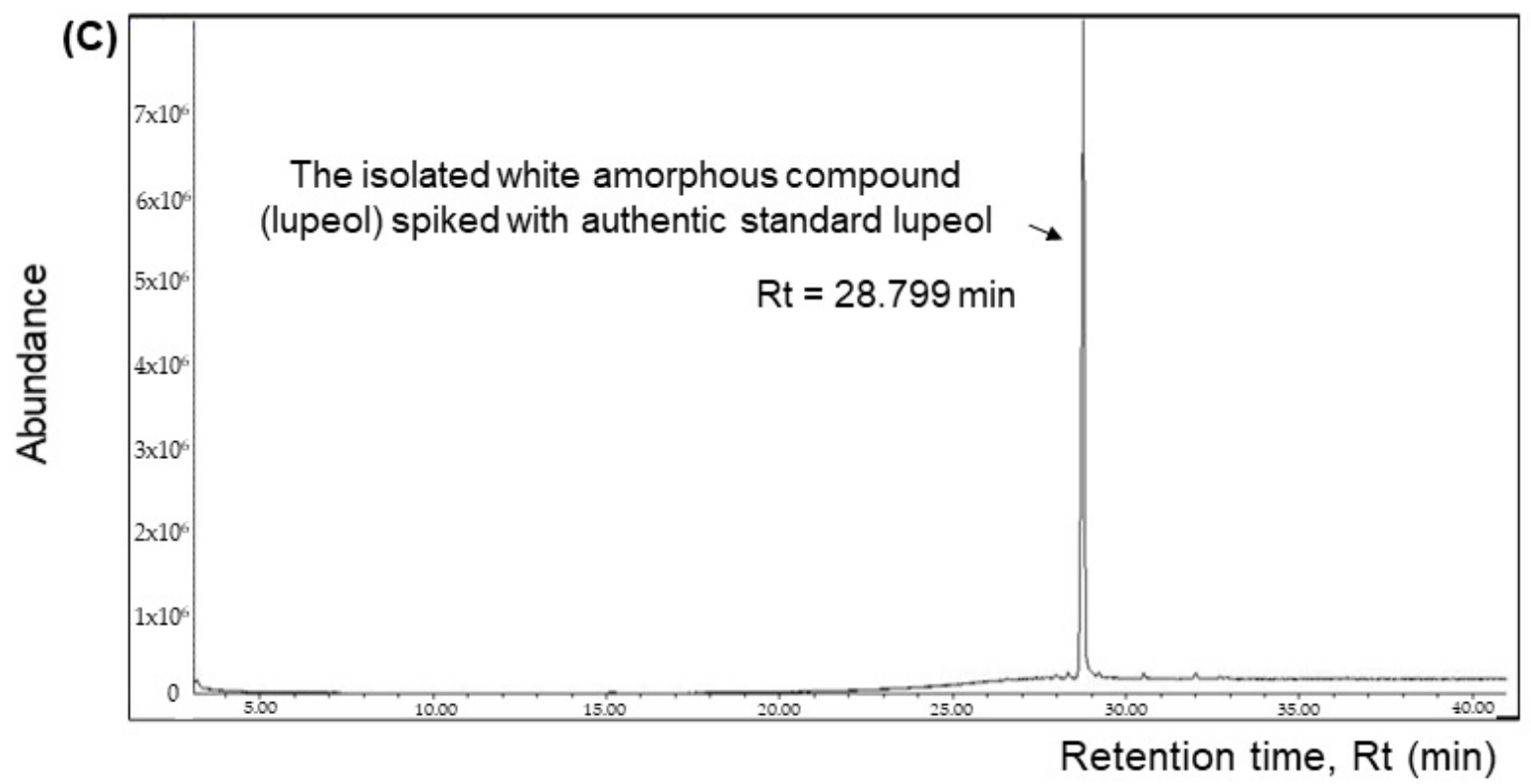

Figure 3. GC-MS profiles of the (A) isolated white amorphous compound from the roots of C. nutans, (B) purchased authentic lupeol, and (C) isolated compound spiked with authentic lupeol. 
Meanwhile, the ${ }^{13} \mathrm{C}-\mathrm{NMR}$ spectrum $\left(150 \mathrm{MHz}, \mathrm{CDCl}_{3}\right)$ of the isolated compound exhibited signals at $\delta 109.36$ (C-29) and $\delta 78.99$ (C-3), which were the characteristics of protons and carbons of a methylene and oxymethine group at the positions 3 and 29 for lupeol [15]. In addition, another distinguishable characteristic of triterpenoid was found at $\delta 150.91$ for the presence of carbon C-20 [15]. The ${ }^{13} \mathrm{C}-\mathrm{NMR}$ also revealed carbon intensity at $\delta 38.86$ (C-1), 27.41 (C-2), 38.74 (C-4), 55.33 (C-5), 18.03 (C-6), 34.31 (C-7), 40.85 (C-8), 50.46(C-9), 37.18 (C-10), 20.95 (C-11), 25.16 (C-12), 38.07 (C-13), 42.84 (C-14), 27.47 (C-15), 35.60 (C-16), 47.99 (C-17), 48.32 (C-18), 47.99(C-19), 29.87 (C-21), 38.86 (C-22), 27.47 (C-23), 15.40 (C-24), 16.13 (C-25), 16.00 (C-26), 14.57 (C-27), 18.34 (C-28), and 19.33 (C-30), which were identical to the reported literature data by $[12,15,16]$. The ${ }^{13} \mathrm{C}-\mathrm{NMR}$ spectrum for the isolated compound can be found in Supplemental Data S1B.

\subsection{The Isolated Lupeol from C. nutans Roots Demonstrates Comparable Anti-proliferative Activity with Authentic Lupeol and Campthothecin}

The anti-proliferative activity of the lupeol isolated from the C. nutans roots was tested against human breast cancer cell line MCF-7 using MTT assay, and the result was compared with the anti-proliferative activity of authentic standard lupeol (Figure 4). The standard lupeol was found to have an $\mathrm{IC}_{50}$ value of $13.624 \pm 1.257 \mu \mathrm{g} / \mathrm{mL}$, whereas the lupeol isolated from C. nutans exhibited a comparable $\mathrm{IC}_{50}$ value of $16.813 \pm 1.316 \mu \mathrm{g} / \mathrm{mL}$. Both authentic and isolated lupeol displayed a potent growth inhibition on MCF-7 cells in a dose-dependent manner (Figure 4). Comparing to camptothecin $\left(\mathrm{IC}_{50}=0.93 \mu \mathrm{g} / \mathrm{mL}\right.$, Supplemental Figure S3), lupeol is about $15 x$ less toxic.

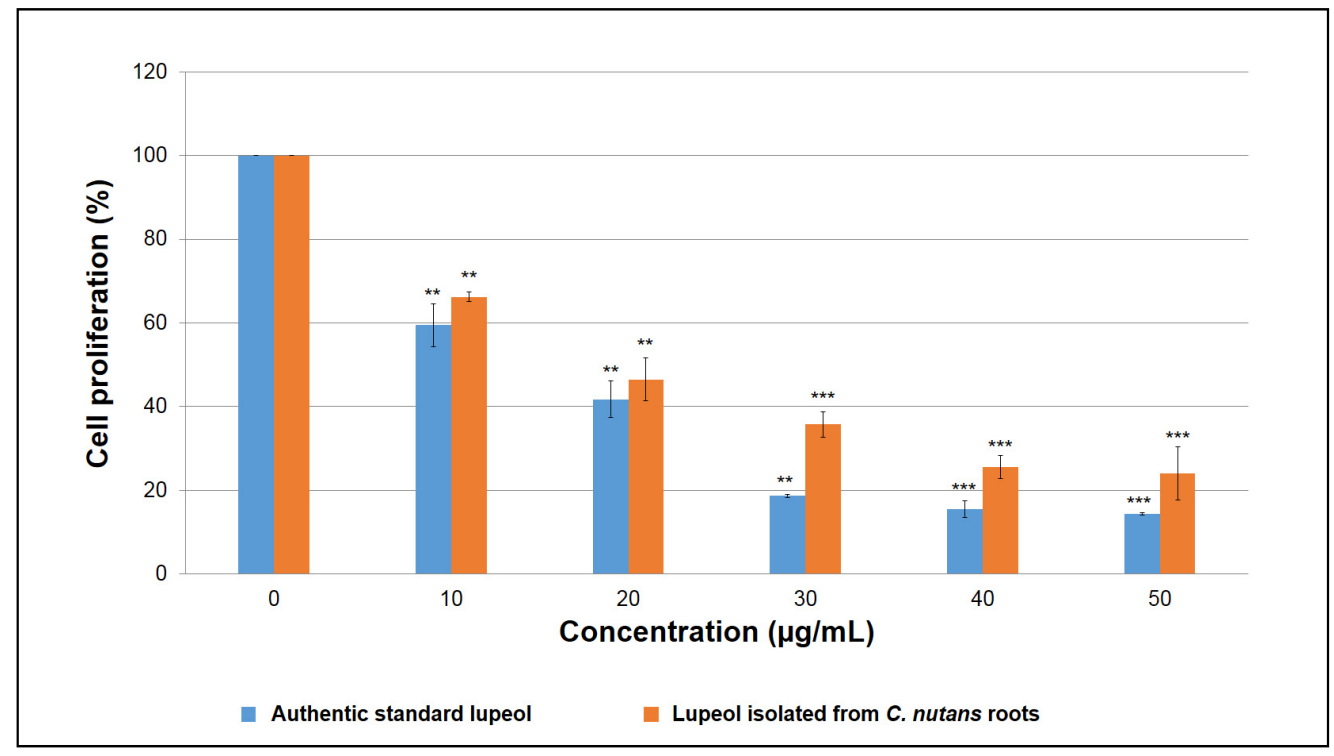

Figure 4. Anti-proliferative activities of the isolated lupeol from C. nutans roots compared to the authentic standard lupeol and camptothecin (Supplemental Figure S3) against MCF-7 cells after $72 \mathrm{~h}$ with the indicated concentrations. The IC 50 values were expressed as mean $\pm \mathrm{SD}$ of the three independent experiments $(n=3)$. Level of significance $\left(^{* *} p<0.01\right.$ and $\left.{ }_{* * *} p<0.001\right)$ for each concentration of authentic or isolated lupeol was compared to the control cells, and was determined using one-way ANOVA followed by a post hoc Dunnett test.

\section{Discussion}

The isolation of anti-proliferative compounds from C. nutans is not easy. This may be due to the complexities of the plant's nature and also various fractionation/chromatographic steps which need to be optimized. We came up with a strategy to pre-screen (profile) the volatile compounds present in every part of the plant; firstly, using a GC-MS method, we have optimized for the detection of volatile compounds [7], then only select the suitable plant part and target compound. In our present study, we found that the aerial part of the plant, i.e., the leaf, was predominated by vitamin $\mathrm{E}$ ( $\alpha$-tocopherol), squalene, and phytos- 
terols such as stigmasterol, $\gamma / \beta$-sitosterol, and campesterol. Interestingly, the intensities of these compounds decreased when moving to the lower part of the plant, i.e., the stem. In the stem, lupeol was found to start to accumulate abundantly and the amount was much higher than squalene and other phytosterols. Lupeol is one of the pentacyclic triterpenes with a 30-carbon skeleton comprising four six-membered rings and one five-membered ring [17]. More intriguingly, when it came to the underground part of the plant, i.e., the root, it was found that this part contained enormous lupeol levels and some betulin. All of the detected phytosterols and pentacyclic terpenoids (lupeol and betulin) in this study have been reported to possess anti-proliferative and apoptotic activities by other researchers [18-27].

Figure 5 illustrates the biosynthesis of phytosterols and some pentacyclic triterpenoids such as lupeol and betulin. The figure was made by referring to [28-30]. The biosynthesis of the compounds begins from the reactions of two precursors called isopentenyl pyrophosphate (IPP) and dimethylallyl pyrophosphate (DMAPP), which are derived from acetyl-CoA via the mevalonic acid (MVA) pathway [28-30]. The reaction is catalyzed by the enzyme isopentenyl pyrophosphate isomerase to yield the geranylpyrophosphate (GPP) and farnesyl pyrophosphate (FPP) via the catalysis of prenyltransferase. Then, two molecules of FPPs are catalyzed by the squalene synthase to yield squalene, which is the important precursor for various phytosterols and pentacyclic triterpenoids. Via the squalene epoxidase, squalene is converted to 2,3-oxidosqualene, which is the branch point for the subsequent biosynthesis of phytosterols or pentacyclic triterpenoids [28-30]. For phytosterols, multiple enzymatic steps are involved in the plants to convert 2,3-oxidosqualene to cycloartenol and end products such as stigmasterol, sitosterol and campesterol [30]. For pentacyclic triterpenoids such as lupeol and betulin, the first step is the conversion of 2,3-oxidosqualene to lupeol by lupeol synthase, and then followed by three sequential oxidations catalyzed by cytochrome P450 monooxygenases (CyP450), leading to the generation of betulin, betulinic aldehyde, and betulinic acid [28,29]. Based on the results observed in our study, it can be postulated that squalene biosynthesis may actively take part in the aerial part (leaf). This squalene then becomes the precursor for the biosynthesis of lupeol in the stem part. Finally, the root part may either become the synthesis site or main storage site for lupeol.

To date, two studies related to the isolation of lupeol from C. nutans plant have been reported. One was reported by Dampawan and colleagues [31] in 1977 in which the plant part used was the stem. Another one was reported by Le and colleagues [15] in 2017 in which the plant parts used were the leaf and stem. Our study is the first report on the isolation of lupeol from the root part. Owing to its high abundance in the roots and simple root profile obtained via GC-MS analysis, we developed a simpler and straight-forward isolation method for lupeol from the plant's roots. In our study, we used an initial $80 \mathrm{~g}$ of freeze-dried root powder and macerated it with ethyl acetate for five days during the first extraction, followed by three days for the second extraction on the root residues with ethyl acetate, and combined the two extracts and dried them. The vacuum-dried extract was re-dissolved in methanol and underwent recrystallization to yield $1.138 \mathrm{~g}$ white amorphous compound, which was identified to be lupeol (yield of extraction $=1.423 \%$ ). In [31], an initial two kilograms of dried and milled C. nutans stems were extracted by using light petroleum in Soxhlet apparatus, followed by silica gel column chromatography using ether and light petroleum for elution, then further purified by using preparative thin layer chromatography and recrystallization to yield $3.7 \mathrm{~g}$ of lupeol (yield $=0.185 \%$ ). In [15], an initial four kilograms of dried and powdered C. nutans leaves were macerated with methanol and filtered, followed by solvent-solvent partitioned with hexane to yield a crude extract of $153.9 \mathrm{~g}$. The crude hexane extract was later dissolved in methanol and chromatographed over Diaion HP20SS using ethanol to yield five sub-fractions ( $\mathrm{H} 1, \mathrm{H} 2, \mathrm{H} 3, \mathrm{H} 4$, and $\mathrm{H} 5)$. These five sub-fractions were further subjected to column chromatography using different gels to produce four pure compounds (shaftoside, stigmasterol, $\beta$-sitosterol, and lupeol). Unfortunately, the final yield for each 
of the pure compounds was not mentioned in the literature and thus could not compare to our method. However, compared to Dampawan, our method manages to produce a higher extraction yield of lupeol. Additionally, our method is simple and straightforward compared to $[15,31]$ as it does not involve solvent-solvent partition, and also without the hassle to go through the column or preparative thin-layer chromatography. The successful isolation of lupeol from C. nutans roots in this study also suggests the importance of GC-MSor LC-MS-based profiling of every part of the plant first prior to the selection of potential candidate compounds for the isolation process.

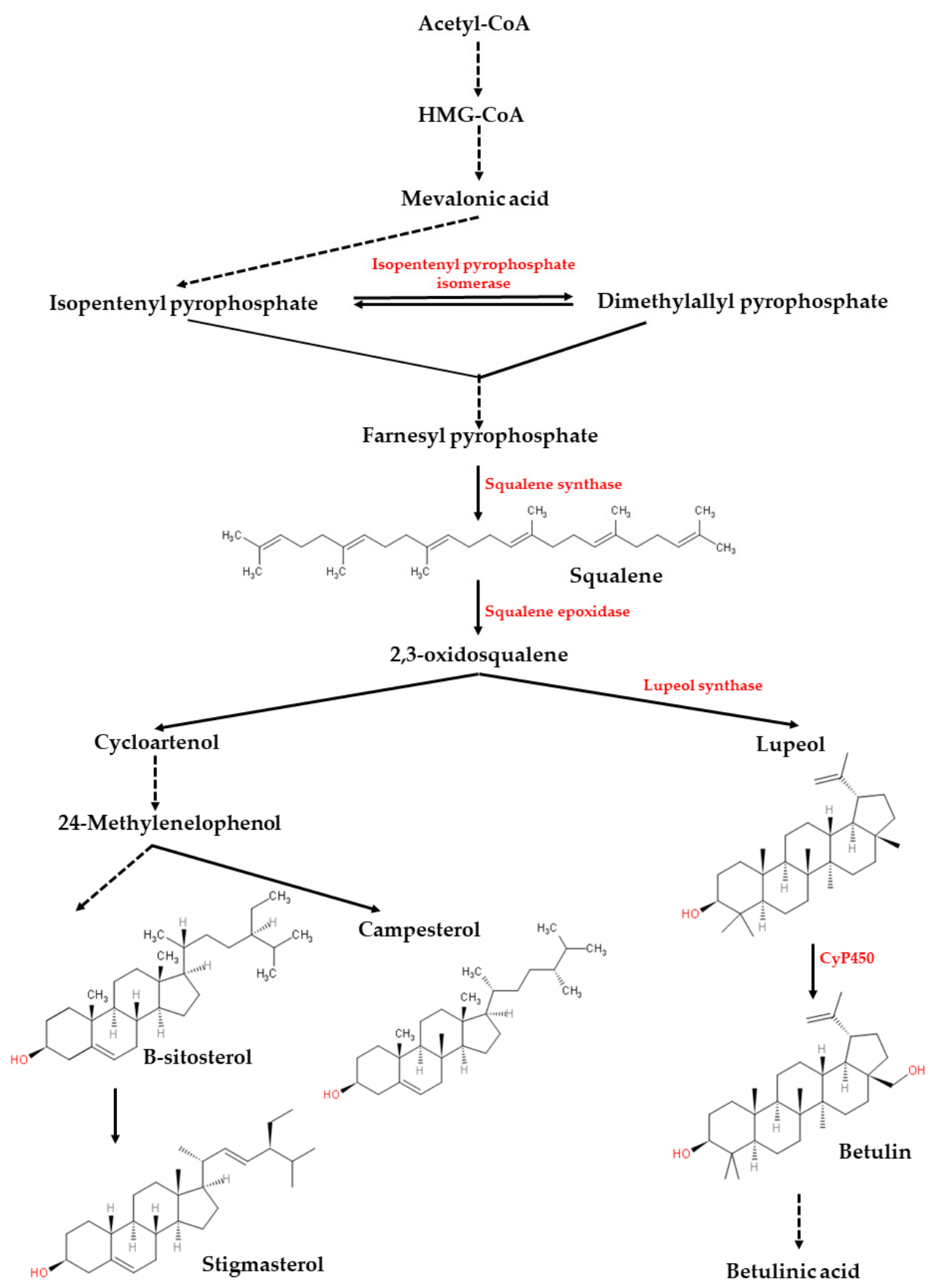

Figure 5. The biosynthesis of pentacyclic terpenoids and phystosterols. The biosynthesis structure and pathway were produced using the KingDraw v2.0 for Windows software (http:/ / www.kingdraw.cn/en/index.html, Qingdao, China. Downloaded and installed on 1 May 2021). 
In addition, the extensive characterization of the isolated compound using UV-visible spectrophotometry, Fourier transform infrared spectroscopy, GC-MS, and nuclear magnetic resonance have been able to identify the isolated compound as lupeol. Further analysis of the anti-proliferative activity of the isolated lupeol against human breast cancer cells has revealed that the isolated lupeol was able to demonstrate comparable anti-proliferative activity with the standard lupeol and positive control camptothecin. A study reported by [22] has postulated the possible mechanism of lupeol on human breast cancer cells by down-regulating the anti-apoptosis proteins, $\mathrm{Bcl}-2$ and $\mathrm{Bcl}-\mathrm{XL}$, which lead to cell apoptosis. Our previous study [7] also shows that the root extract from C. nutans is capable of causing apoptosis of the breast cancer cells via the condensation of chromatin, down-regulation of the BCL2 gene, and the loss of mitochondrial membrane potential of the cancer cells. We therefore highly speculate that the pro-apoptotic capability of $C$. nutans roots could be highly correlated with the lupeol contained inside the roots. We therefore are interested in exploring the molecular mechanisms associated with anti-proliferative activity exerted by the isolated lupeol. Further, the profiling method presented in this study is a simple qualitative study of volatile compounds in different parts of $C$. nutans to aid in the isolation of the anti-proliferative compound. Referring to the successful GC-MS-based metabolomics study of $C$. nutans leaf extracts on $\alpha$-glucosidase activity as reported by [32], we are also interested in applying this similar sophisticated GC-MS-based metabolomics in the future to explore the anti-proliferative mechanism of lupeol isolated from C. nutans plants harvested from different growing stages or collected from different regions.

\section{Conclusions}

In conclusion, comparing the plant's volatile profiles greatly narrowed down our target of study. Our strategy to profile every part of the plant first, followed by selection of most suitable plant's part and target compound are useful for further isolation and characterization of anti-proliferative compounds such as lupeol from the roots of $C$. nutans.

Supplementary Materials: The following are available online at https:/ /www.mdpi.com/article/10 .3390/pr9081383/s1: Figure S1: The electron ionization-mass spectrum (EI-MS) of the (A) isolated and (B) authentic lupeol; Figure S2: Fourier-transform infrared spectrum (FTIR) of the white amorphous compound (lupeol) isolated from C. nutans roots; Figure S3: Cytotoxicity assay of camptothecin against MCF-7 cell lines; Supplemental Data S1A: 1H-NMR spectrum of the isolated compound from C. nutans roots; Supplemental Data S1B: 13C-NMR spectrum of the isolated compound from C. nutans roots.

Author Contributions: Conceptualization, P.L.T. and B.E.C.; methodology, A.Y.F.C.; validation, A.Y.F.C., B.E.C., L.J. and P.L.T.; formal analysis, investigation and writing—original draft preparation, A.Y.F.C.; writing - review and editing, B.E.C., L.J. and P.L.T.; visualization, A.Y.F.C.; supervision, B.E.C. and P.L.T.; project administration, P.L.T.; funding acquisition, B.E.C. and P.L.T. All authors have read and agreed to the published version of the manuscript.

Funding: This research was funded by Universiti Malaysia Sabah (UMS) Research Grant Scheme, grant number SBK0070-SG-2013 and the Malaysia Ministry of Education, grant number FRG0360-SG-2/2013.

Acknowledgments: The authors thank Mohd. Zahid Md. Yusuff from the Universiti Kebangsaan Malaysia (UKM), Malaysia for the assistance of running the NMR experiments in this study.

Conflicts of Interest: The authors declare no conflict of interest.

\section{References}

1. Chelyn, J.L.; Omar, M.H.; Yousof, N.S.A.; Ranggasamy, R.; Wasiman, I.; Ismail, Z. Analysis of flavone C-glycosides in the leaves of Clinacanthus nutans (Burm. F.) Lindau by HPTLC and HPLC-UV/DAD. Sci. World. J. 2014, 2014, 1-6. [CrossRef]

2. Nik Abd Rahman, N.M.A.; Nurliyana, M.Y.; Nur Afiqah, M.N.F.N.; Osman, N.M.; Hamid, N.; Mohd Lila, M.A. Antitumor and antioxidant effects of Clinacanthus nutans Lindau in 4 T1 tumor-bearing mice. BMC Complement. Altern. Med. 2019, 19, 340. [CrossRef] 
3. Yong, Y.K.; Tan, J.J.; Teh, S.S.; Mah, S.H.; Cheng, G.L.E.; Chiong, H.S.; Ahmad, Z. Clinacanthus nutans extracts are antioxidant with antiproliferative effect on cultured human cancer cell lines. Evid. Based Complement. Altern. Med. 2013, 2013, 462751. [CrossRef] [PubMed]

4. Che Sulaiman, I.S.; Basri, M.; Chan, K.W.; Ashari, A.E.; Masoumi, H.R.F.; Ismail, M. In vitro antioxidant, cytotoxic and phytochemical studies of Clinacanthus nutans Lindau leaf extracts. Afr. J. Pharm. Pharmacol. 2015, 9, 861-874. [CrossRef]

5. Fong, S.Y.; Piva, T.; Dekiwadia, C.; Urban, S.; Huynh, T. Comparison of cytotocity between extracts of Clinacanthus nutans (Burm. f.) Lindau leaves from different locations and the induction of apoptosis by the crude methanol leaf extract in D24 human melanoma cells. BMC Complement. Altern. Med. 2016, 16, 368. [CrossRef] [PubMed]

6. Huang, D.; Guo, W.; Gao, J.; Chen, J.; Olatunji, J.O. Clinacanthus nutans (Burm. f.) Lindau ethanol extract inhibits hepatoma in mice through upregulation of the immune response. Molecules 2015, 20, 17405-17428. [CrossRef] [PubMed]

7. Teoh, P.L.; Cheng, A.Y.F.; Liau, M.; Lem, F.F.; Kaling, G.P.; Chua, F.N.; Cheong, B.E. Chemical composition and cytotoxic properties of Clinacanthus nutans root extracts. Pharm. Biol. 2017, 55, 394-401. [CrossRef]

8. Huang, D.; Li, Y.; Cui, F.; Chen, J.; Sun, J. Purification and characterization of a novel polysaccharide-peptide complex from Clinacanthus nutans Lindau leaves. Carbohydr. Polym. 2016, 137, 701-708. [CrossRef] [PubMed]

9. Mutazah, R.; Hamid, H.A.; Mazila Ramli, A.N.; Fasihi Mohd Aluwi, M.F.; Yusoff, M.M. In vitro cytotoxicity of Clinacanthus nutans fractions on breast cancer cells and molecular docking study of sulphur containing compounds against caspase-3. Food Chem. Toxicol. 2020, 135, 110869. [CrossRef]

10. Agidew, E.; Reneela, P.; Tsegaye, D. Phytochemical investigation of Sapium elliptum. J. Nat. Prod. Plant Res. 2013, 3, 1-6.

11. Pereira, F.B.M.; Domingues, F.M.J.; Silva, A.M.S. Triterpenes from Acacia dealbata. Nat. Prod. Lett. 1996, 8, 97-103. [CrossRef]

12. Prachayasittikul, S.; Saraban, P.; Cherdtrakulkiat, R.; Ruchirawat, S.; Prachayasittikul, V. New bioactive triterpenoids and antimalarial activity of Diospyros rubra Lec. EXCLI J. 2010, 9, 1-10.

13. Saratha, V.S.; Pillai, I.; Subramaniam, S. Isolation and characterization of lupeol, a triterpenoid from Calotropis gigantea latex. Int. J. Pharm. Sci. Rev. Res. 2011, 10, 54-57.

14. Hague, M.A.; Hassan, M.M.; Das, A.; Begum, B.; Ali, M.Y.; Morshed, H. Phytochemical investigation of Veronia cinerea (Family: Asteraceae). J. Appl. Pharm. Sci. 2012, 2, 79-83. [CrossRef]

15. Le, C.-F.; Kailaivasan, T.H.; Chow, S.-C.; Abdullah, Z.; Ling, S.-K.; Fang, C.-M. Phytosterols isolated from Clinacanthus nutans induce immunosuppressive activity in murine cells. Int. Immunopharm. 2017, 44, 203-210. [CrossRef] [PubMed]

16. Ragasa, C.Y.; Tan, M.C.S.; Fortin, D.R.; Shen, C.-C. Chemical constituents of Ixora philippinensis Merr. J. Appl. Pharm. Sci. 2015, 5, 62-67. [CrossRef]

17. Saleem, M.; Kaur, S.; Kweon, M.-H.; Adhami, V.M.; Afaq, F.; Mukhtar, H. Lupeol, a fruit and vegetable based triterpene, induces apoptotic death of human pancreatic adenocarcinoma cells via inhibition of Ras signaling pathway. Carcinogenesis 2005, 26, 1956-1964. [CrossRef] [PubMed]

18. Lee, Y.S.; Tee, C.T.; Tan, S.P.; Awang, K.; Hashim, N.M.; Nafiah, M.A.; Ahmad, K. Cytotoxic, antibacterial and antioxidant activity of triterpenoids from Kopsia singapurensis Ridl. J. Chem. Pharm. Res. 2014, 6, 815-822.

19. Saleem, M.; Kweon, M.-H.; Yun, J.-M. A novel dietary triterpene lupeol induces fas-mediated apoptotic death of androgensensitive prostate cancer cells and inhibits tumor growth in a xenograft model. Cancer Res. 2005, 65, 11203-11213. [CrossRef]

20. Saleem, M. Lupeol, a novel anti-inflammatory and anti-cancer dietary triterpene. Cancer Lett. 2009, 285, 109-115. [CrossRef]

21. Saleem, M.; Murtaza, I.; Tarapore, R.S.; Suh, Y.; Adhami, V.M.; Johnson, J.J.; Siddiqui, I.A.; Khan, N.; Asim, M.; Hafeez, B.B.; et al. Lupeol inhibits proliferation of human prostate cancer cells by targeting $\beta$-catenin signaling. Carcinogen 2019, 30, 808-817. [CrossRef]

22. Pitchai, D.; Roy, A.; Ignatius, C. In vitro evaluation of anticancer potentials of lupeol isolated from Elephantopus scaber L. on MCF-7 cell line. J. Adv. Pharm. Technol. Res. 2014, 5, 179-184. [CrossRef]

23. Kommera, H.; Kaluderovic, G.N.; Kalbitz, J.; Paschke, R.P. Lupane triterpenoids-Betulin and betulinic acid derivatives induce apoptosis in tumor cells. Investig. New Drugs 2011, 29, 266-272. [CrossRef]

24. Awad, A.B.; Williams, H.; Fink, C.S. Effect of phytosterols on cholesterol metabolism and MAP kinase in MDA-MB-231 human breast cancer cells. J. Nutr. Biochem. 2003, 14, 111-119. [CrossRef]

25. Ghosh, T.; Maity, T.K.; Singh, J. Evaluation of antitumor activity of stigmasterol, a constituent isolated from Bacopa monnieri Linn aerial parts against Ehrlich Ascites carcinoma in mice. Orient. Pharm. Exp. Med. 2011, 11, 41-49. [CrossRef]

26. Chai, J.W.; Kuppusamy, U.R.; Kanthimathi, M.S. beta-Sitosterol induces apoptosis in MCF-7 cells. Malays. J. Biochem. Mol. Biol. 2008, 16, 28-30.

27. Sundarraj, S.; Thangam, R.; Sreevani, V.; Kaveri, K.; Gunasekaran, P.; Achiraman, S.; Kannan, S. $\gamma$-Sitosterol from Acacia nilotica L. induces G2/M cell cycle arrest and apoptosis through c-Myc suppression in MCF-7 and A549 cells. J. Ethnopharmacol. 2012, 141, 803-809. [CrossRef]

28. Jin, C.-C.; Zhang, L.-L.; Song, H.; Cao, Y.-X. Boosting the biosynthesis of betulinic acid and related triterpenoids in Yarrowia lipolytica via multimodular metabolic engineering. Microb. Cell Fact. 2019, 18, 77. [CrossRef]

29. Majumder, S.; Ghosh, A.; Bhattacharya, M. Natural anti-inflammatory terpenoids in Camellia japonica leaf and probable biosynthesis pathways of the metabolome. Bull. Natl. Res. Centre 2020, 44, 141. [CrossRef]

30. Piironen, V.; Lindsay, D.G.; Miettinen, T.A.; Toivoi, J.; Lampi, A.-M. Plant sterols: Biosynthesis, biological function and their importance to human nutrition. J. Sci. Food Agric. 2000, 80, 939-966. [CrossRef] 
31. Dampawan, P.; Huntrakul, C.; Reutrakul, V. Constituents of Clinacanthus nutans and the crystal structure of lup-20(29)-ene-3-one. J. Sci. Soc. Thail. 1977, 3, 14-26. [CrossRef]

32. Murugesu, S.; Ibrahim, Z.; Ahmed, Q.-U.; Nik Yusoff, N.-I.; Uzir, B.-F.; Perumal, V.; Abas, F.; Saari, K.; El-Seedi, H.; Khatib, A. Characterization of $\alpha$-Glucosidase Inhibitors from Clinacanthus nutans Lindau Leaves by Gas Chromatography-Mass SpectrometryBased Metabolomics and Molecular Docking Simulation. Molecules 2018, 23, 2402. [CrossRef] [PubMed] 